\title{
Politique
}

Politique

\section{Louise Vandelac, Diane Bélisle, Anne Gauthier, Yolande Pinard, Du travail et de l'amour. Les dessous de la production domestique, Montréal, Saint-Martin, 1985, 418 p.}

\section{Denyse Côté}

Numéro 8, automne 1985

Innovations et politiques technologiques

URI : https://id.erudit.org/iderudit/040504ar

DOI : https://doi.org/10.7202/040504ar

Aller au sommaire du numéro

Éditeur(s)

Société québécoise de science politique

ISSN

0711-608X (imprimé)

1918-6584 (numérique)

Découvrir la revue

Citer ce compte rendu

Côté, D. (1985). Compte rendu de [Louise Vandelac, Diane Bélisle, Anne Gauthier, Yolande Pinard, Du travail et de l'amour. Les dessous de la production domestique, Montréal, Saint-Martin, 1985, 418 p.] Politique, (8), 155-159. https://doi.org/10.7202/040504ar d'utilisation que vous pouvez consulter en ligne.

https://apropos.erudit.org/fr/usagers/politique-dutilisation/ 
Louise Vandelac, Diane Bélisle, Anne Gauthier, Yolande Pinard, Du travail et de l'amour. Les dessous de la production domestique, Montréal, Saint-Martin, 1985, 418 p.

«La majorité des hommes paient pour leur hétérosexualité, alors que la plupart des femmes en vivent» (p. 337)

Voilà campée la contingence pour les femmes du procès de travail domestique, maintenue, entretenue par «les écarts de revenus hommes-femmes ainsi que (par) l'ensemble de l'organisation sociale». Envers du travail salarié ou rémunérateur, ce procès de travail est aussi l'envers de l'amour, car il suppose l'existence d'une assymétrie dans les rapports amoureux hétérosexuels. Voici enfin arrivée la publication tant attendue de ce livre, version remaniée d'une recherche préparatoire au FORUM économique des femmes convoqué en 1983 par le Conseil du statut de la femme. L'initiative de publication par les éditions coopératives St-Martin est très heureuse, car elle rend ainsi de nouveau accessible cette première étude québécoise aussi exhaustive de la question, dont l'édition originale (par le CSF) avait rapidement été épuisée. 
L'intérêt de ce livre, qui reprend et développe dans le contexte académique québécois un débat déjà amorcé en Angleterre, en France et aux États-Unis par les Oakley, Delphy, Ehrenreich et autres, est de rendre accessible aux non-initiés tout un corpus bibliographique et une synthèse des débats en cours sur le sujet. Le grand public aura en main un outil de travail québécois, qui souligne les tenants et aboutissants de la reproduction domestique, mais aussi du débat politique qui l'entoure ainsi que des propositions ou stratégies de transformation mises de l'avant par différents courants.

Plus précisément, le livre étudie l'articulation de la reproduction domestique avec la reproduction marchande: on se penche et sur le travail salarié des femmes, et sur le travail domestique. Reproduction domestique qui, certes, modèle la vie de toutes les femmes, indépendamment de leur situation particulière, mais surtout qui «constitue, en fait, la base et la face cachée de l'actuel système économique et social» (p. 13). Intéressante tentative d'analyser l'économie politique d'un phénomène généralisé mais non-reconnu, et dont les manifestations sont si variées. On a voulu pour la circonstance (heureuse initiative!), sortir de l'association mécanique entre le concept de reproduction domestique et la situation de mère de famille économiquement dépendante et hors emploi toute sa vie, «modèle en régression, de plus en plus fluctuant et bardé d'exceptions». Les auteures se sont au contraire ouvertes à la réalité multiforme, pour inclure les travailleuses à domicile, les femmes collaboratrices, les travailleuses salariées, les mères sans conjoint, les femmes au foyer par intermittance, les femmes «modernes», qui croyaient «qu'en le désertant, le monde domestique s'effilocherait et que le modèle de femme au foyer s'évanouirait dans l'emploi et la libération sexuelle» (p. 12), et, enfin, les femmes actuellement sans conjoint et sans enfant à charge, mais dont «La vie a été consacrée, ou du moins modelée, par la reproduction domestique» (idid.). 
Il s'agit d'un livre imbu d'une même problématique, mais construit comme «reader» ou colligé d'articles. Dans un premier temps, Louise Vandelac nous dresse un portrait de la problématique de la reproduction domestique, se référant pour ceci à l'histoire, l'économie, à l'analyse sociologique: elle campe le sujet du livre en débusquant les dessous de travail salarié, elle reprend le débat sur les stratégies revendicatrices, dont celle du «salaire contre le travail domestique». Dans la section suivante, Diane Belisle et Yolande Pinard brossent en deux articles un tableau historique du travail domestique, tant au Québec qu'en France, en Angleterre et aux États-Unis. On y souligne par exemple que si à la période pré-industrielle en Angleterre les femmes, "aussi subalternes soientelles, ne sont pas réduite à l'état de servantes, sans pouvoir aucun » (p. 71), ce n'est pas l'industrialisation en tant que telle qui a exclu les femmes de la production marchande. En effet, selon les auteures, «dans la deuxième moitié du $\mathrm{XIX}^{\mathrm{e}}$ siècle, les employeurs sont sans doute les moins convaincus qu'une ouvrière vaut moins qu'un ouvrier: pour eux, elle revient moins chère, tolère mieux la fatigue, les longues heures de travail, la monotonie, la discipline, etc» (p. 75). C'est plutôt l'idéologie et la pratique du salaire familial qui ont vu à leur exclusion, pratique selon laquelle le salaire ouvrier devrait suffire à faire vivre sa famille. L'article portant sur le Québec raconte grosso modo la même histoire, truffée cependant d'exemples imagés et pertinents sur l'organisation quotidienne des québécoises.

Suit une très intéressante réflexion de Diane Bélisle sur le temps domestique versus le temps marchand, où l'auteure analyse leur mutuelle incompatibilité. Elle recense les diverses écoles qui ont analysé le temps domestique et critique celles qui, comme les tenants de la technique du budget-temps, "(scrutent) à la loupe le phénomène domestique... au regard d'un temps-argent, d'un temps marchand». Par la suite, Louise Vandelac nous livre un texte (plus serré que son premier), sur les évaluations monétaires 
du travail domestique. Les approches et méthodes de calcul y sont exposées en détail et analysées systématiquement: avis aux intéressées (és). Elle termine l'article par l'analyse du concept de l'homo œeconomicus, cet «homme solitaire et calculateur, à la poursuite incessante de ses propres intérêts... (et qui) évacue les questions relatives à la reproduction non marchande, à croire qu'il se reproduit par miracle économique» (p. 241). Cet homo œconimicus, imposé comme normalité, a créé l'anormalité et l'anachronisme des femmes chargées par défaut de la reproduction domestique.

Par la suite, l'article d'Anne Gauthier porte sur les politiques sociales en matière de la reproduction domestique. Bien recherché, bien structuré il s'attele à l'histoire de différentes politiques sociales telles l'aide aux mères nécessiteuses, l'aide sociale. Enfin, le dernier article du livre porte sur le partage des tâches domestiques: il aborde la face voilée de ce nouvel égalitarisme apparent; il décortique les approches féminines et masculines du partages des tâches. À ce titre, on y souligne une profonde différence au niveau du temps fournit, du type de tâches effectuées, du rapport de l'individu selon le sexe à la reproduction domestique (s'agit-il de différentier les niveaux de responsabilisation?)

Le livre pèche cependant par sa longueur et son inégalité: les répétitions des mêmes thèmes sont nombreuses, et, quoique développés de façon originale dans chaque cas auraient mérité des renvois, ou des choix: fondre les deux articles historique ou en choisir un seul (ils sont, chacun en soi, intéressants). L'analyse du travail salarié vs le travail domestique est souvent reprise, et risque de finir par ennuyer. Bref, on y voit l'intention de vulgariser les débats à travers une analyse académique, mais l'effort de vulgarisation et de synthèse semble être resté en friche. Aurionsnous, à ce titre-là, été gâtées par l'excellente expérience de vulgarisation du Collectif Clio (Histoire des femmes au Québec), Quinze, 1982)? 
Le premier article du livre, qui devrait circonscrire l'objet d'étude, est décevant. Les idées sont originales, diverses, mais le tout laisse l'impression d'un article en chantier, tant au niveau de la structure interne de l'article, que de la rigueur théorique. Malgré l'avertissement de l'auteure sur la difficile définition de l'objet, avis que je partage, il est difficilement acceptable de se contenter d'une définition poétique et économique d'un objet pourtant abordé comme profondément sociologique et politique. À titre d'exemple, qu'est-ce qui pourrait justifier l'interchangeabilité presque mécanique des concepts de maternité, maternage, reproduction domestique, activité domestique, production domestique, question domestique, travail domestique? On note par ailleurs une nette tendance à cerner l'objet par le biais de données empiriques: serait-ce là la source de ces hésitations ou vides théoriques?

Une autre faiblesse du livre que je me dois de relever dans les circonstances, est la faiblesse au niveau de l'analyse politique. Affirmer que «les analyses de fond ainsi que le travail de critique épistémologique du champ politique (par les féministes) ne sont que balbutiantes» (p. 67), ce dont je ne suis pas du tout convaincue, ne justifie pas ce vide. Nos espoirs à la lecture du titre de chapitre «État-mari, État-papa», sont vite déçus. Écrit par une économiste de profession, on en reste encore là trop près des faits et du déroulement de l'histoire des politiques sociales; on s'aventure peu sur le terrain de l'analyse politique. Par ailleurs, et en dernier lieu, la volonté clairement exprimée par la coordonatrice du livre de mener le débat politique sur la question, mène parfois à des harangues ou à des affirmations presque moralisatrices qui ne peuvent tenir lieu d'analyse politique.

Bref un livre à lire, mais en pièces détachées, puisqu'il constitue le seul livre en son genre au Québec, généralement accessible et compilant données québécoises et débats internationaux sur la reproduction domestique.

Denyse Côté

Université de Montréal 